\title{
Effetto del radioiodio sulla qualità di vita a lungo termine nei pazienti guariti dal Morbo di Graves-Basedow
}

\author{
Maria Laura Tanda ${ }^{1}$
}

Accettato: 10 agosto 2020 / Pubblicato online: 30 ottobre 2020

(c) The Author(s) 2020

Commento a:

Impaired quality of life after radioiodine therapy compared with antithyroid drugs or surgical treatment for Graves' hyperthyroidism: a long-term follow-up with the Thyroid-Related Patient-Reported Outcome questionnaire and 36-Item Short Form Health Status Survey.

O. Törring, T. Watt, G. Sjölin, K. Byström,

M. Abraham-Nordling, J. Calissendorff, P.K. Cramon,

H. Filipsson Nyström, B. Hallengren, M. Holmberg,

S. Khamisi, M. Lantz, G. Wallin.

Thyroid (2019) 29:322-331

La malattia di Graves determina, nei pazienti affetti, una riduzione significativa della qualità della vita determinata da vari fattori [1,2]. Questa riduzione può essere dovuta all'intensità dell'ipertiroidismo, generalmente maggiore rispetto a ipertiroidismi di altra natura, alla presenza di orbitopatia che causa alterazioni della fisionomia, alla slatentizzazione di manifestazioni psichiatriche che si possono associare alla malattia di Graves. Anche la concomitanza di altre patologie autoimmuni può concorrere alla riduzione della qualità di vita effettiva o percepita.

I cardini del trattamento dell'ipertiroidismo di Graves sono rappresentati dal trattamento conservativo medico con antitiroidei di sintesi oppure dal trattamento definitivo chirurgico o con radioisotopi dello iodio. Studi anche randomizzati ma condotti su casistiche relativamente piccole ed eseguiti a breve distanza dal trattamento non hanno mostrato significative differenze di qualità di vita in funzione delle

M.L. Tanda

marialaura.tanda@uninsubria.it

1 Dipartimento di Medicina e Chirurgia, Unità di Endocrinologia, Università degli Studi dell'Insubria, Varese, Italia tre modalità di trattamento. La valutazione degli effetti percepiti dal paziente in relazione alla malattia e al suo trattamento viene valutata attraverso questionari validati sia di tipo generico sia di tipo specifico (es. FS-36, ThyPro, GoQol, TED-QoL) [3].

In questo studio gli autori si sono proposti di valutare, su un campione ampio di pazienti trattati secondo le regole della pratica clinica, la qualità di vita $(\mathrm{Q} o \mathrm{~L})$ percepita a lunga distanza dalla correzione della malattia. A tale scopo è stata arruolata una coorte di 1.976 pazienti con prima diagnosi di Graves, afferiti presso sette centri svedesi, durante il periodo 2003-2005; a tutti i pazienti sono stati forniti due questionari SF-36 e Thyr-Pro. Dei soggetti reclutati, una piccola quota non ha aderito allo studio mentre un gruppo di 545 soggetti non ha poi restituito i questionari.

Sono state ottenute le risposte di 1.186 pazienti reclutati e valutati a distanza di un intervallo di 6-10 anni dal trattamento e poi esclusi i pazienti ancora non in remissione di malattia. I questionari pervenuti sono stati in misura lievemente superiore per Thyr-Pro rispetto alle risposte ottenute con SF36 (975 vs 964). Il campione di risposte è stato suddiviso in tre gruppi: il primo di pazienti trattati con soli antitiroidei di sintesi (374), il secondo di pazienti trattati con radioiodio (395) e il terzo di pazienti trattati con tiroidectomia (233), di cui alcuni sottoposti in precedenza a trattamento con radioiodio. La media del follow-up è risultata 8 anni dalla diagnosi di malattia.

Il gruppo trattato con radioiodio differiva per età maggiore (mediana 54 anni), rispetto al gruppo trattato con antitiroidei (mediana 43 anni) e al gruppo sottoposto a chirurgia (mediana 35 anni) e per maggiore numero di comorbidità, mentre non vi erano significative differenze di distribuzione per sesso. Questo in linea con le indicazioni cliniche generalmente seguite nella scelta del trattamento. I pazienti che non hanno risposto ai questionari differivano per età più gio- 
vane (circa 4 anni), per maggior provenienza extraeuropea e per una quota minore di pazienti trattati con radioiodio.

L'analisi dei questionari ha confermato che la malattia di Graves si associa a una riduzione significativa nel lungo termine della qualità della vita rispetto alla popolazione generale, anche dopo un efficace trattamento.

I soggetti sottoposti a radioiodio avevano infatti punteggi inferiori in tutte le scale rispetto alla popolazione generale, mentre i pazienti trattati con antitiroidei o con chirurgia avevano score ridotti in molti dominii ma simili alla popolazione generale.

Nell'analisi dei sottogruppi il trattamento con radioiodio mostrava correlazione con punteggi inferiori in quasi tutti i dominii (per entrambi i questionari), sia rispetto alla terapia medica conservativa, sia rispetto alla tiroidectomia, con differenze maggiori relative alla compromissione della vita sessuale e a sintomi fisici nei pazienti sottoposti a radioiodio rispetto ai pazienti trattati farmacologicamente.

Rispetto ai pazienti tiroidectomizzati, le risposte dei pazienti trattati con terapia radiometabolica mostravano maggior compromissione delle scale correlate a sintomi fisici e psichici (ansia e depressione); inoltre, nel questionario generale SF-36 si sono registrati, nel gruppo radioiodio, punteggi inferiori in tutte le scale (più evidenti per salute generale, funzione sociale e limitazioni generali) eccetto che per il dolore fisico maggiore nei pazienti operati.

Lo studio eseguito in un'ampia popolazione di pazienti basedowiani fornisce ulteriore conferma dell'impatto negativo a lungo termine sulla qualità della vita di questi pazienti rispetto alla popolazione generale, confermando i risultati derivanti da una casistica più contenuta, studiata dagli stessi autori, con un follow-up di 14-21 anni [4].

Tali effetti negativi sembrano correlabili alle conseguenze psichiche e somatiche tipiche di questa forma di ipertiroidismo, in buona parte attribuibili alla presenza di orbitopatia ma forse, come recentemente ripreso alcuni autori, anche al rischio di sovrappeso secondario alla correzione dell'ipertiroidismo, che si ipotizza direttamente correlato alla gravità/durata della tireotossicosi $[5,6]$.

Riguardo, invece, agli effetti derivanti dalla scelta terapeutica, i risultati dello studio appaiono parzialmente in contrasto con precedenti studi, alcuni randomizzati, anche dello stesso gruppo di autori, dove il tipo di trattamento dell'ipertiroidismo era apparso, in termini di QoL, sostanzialmente ininfluente, perlomeno nella valutazione a breve termine [79]. Motivi di questa apparente discrepanza potrebbero essere legati in parte alla casistica di studi precedenti con followup a più breve termine; infatti, in uno studio randomizzato di pazienti suddivisi per età, i pazienti trattati con anti-tiroidei presentavano elevato tasso di recidive e i pazienti sottoposti a radioiodio presentavano maggior frequenza di sintomi oculari [8]; un successivo studio randomizzato con followup a 4 anni mostrava risultati sovrapponibili confermando che la riduzione della QoL è correlata allo sviluppo di orbitopatia; tuttavia, in questo caso il tipo di controllo biochimico a lungo termine e la storia naturale dell'orbitopatia possono condizionare maggiormente la QoL rispetto al tipo di trattamento utilizzato [9].

L'ipotesi che la scelta della terapia dell'ipertiroidismo possa determinare effetti sul paziente, indipendentemente dalla guarigione dall'ipertiroidismo, rende necessari studi di conferma e la ricerca degli eventuali meccanismi sottostanti. In ogni caso, questi risultati stimolano la riflessione che potrebbe avere influenza in futuro sul comportamento da adottare nella gestione di malattia. Se da una parte i dati consolidati della letteratura sulla sicurezza del radioiodio nella cura dell'ipertiroidismo e l'evidenza pratica che la scelta verso il radioiodio sia condizionata da fattori contingenti, quali l'età più avanzata e/o presenza di maggiori comorbidità, che di per sé condizionano in maniera rilevante la QoL del paziente, la possibilità che il radioiodio possa determinare effetti a lungo termine sull'organismo, agendo attraverso meccanismi come le modalità di sviluppo dell'ipotiroidismo, o altri solo ipotizzabili come, ad esempio, l'effetto su trasportatori dello iodio anche differenti da NIS, alcuni recentemente identificati in vari tessuti extratiroidei [10], deve essere approfondita ed eventualmente considerata nell'approccio clinico a questa malattia.

Funding Note Open access funding provided by Università degli Studi dell'Insubria within the CRUI-CARE Agreement.

Nota della casa editrice Springer Nature rimane neutrale in riguardo alle rivendicazioni giurisdizionali nelle mappe pubblicate e nelle affiliazioni istituzionali.

Open Access This article is licensed under a Creative Commons Attribution 4.0 International License, which permits use, sharing, adaptation, distribution and reproduction in any medium or format, as long as you give appropriate credit to the original author(s) and the source, provide a link to the Creative Commons licence, and indicate if changes were made. The images or other third party material in this article are included in the article's Creative Commons licence, unless indicated otherwise in a credit line to the material. If material is not included in the article's Creative Commons licence and your intended use is not permitted by statutory regulation or exceeds the permitted use, you will need to obtain permission directly from the copyright holder. To view a copy of this licence, visit http://creativecommons.org/licenses/by/4.0/.

\section{Bibliografia}

1. Elberling TV, Rasmussen AK, Feldt-Rasmussen U et al (2004) Impaired health-related quality of life in Graves' disease. A prospective study. Eur J Endocrinol 151(5):549-555

2. Riguetto CM, Neto AM, Tambascia MA, Zantut-Wittmann DE (2019) The relationship between quality of life, cognition, and thyroid status in Graves' disease. Endocrine 63(1):87-93

3. Watt T, Groenvold M, Rasmussen AK et al (2006) Quality of life in patients with benign thyroid disorders. A review. Eur $\mathrm{J}$ Endocrinol 154(4):501-510 
4. Abraham-Nordling M, Törring O, Hamberger B et al (2005) Graves' disease: a long-term quality-of-life follow up of patients randomized to treatment with antithyroid drugs, radioiodine, or surgery. Thyroid 15(11):1279-1286

5. Kyriacou A, Kyriacou A, Makris KC et al (2019) Weight gain following treatment of hyperthyroidism-A forgotten tale. Clin Obes 9(5):e12328

6. Alonso GT, Rabon S, White PC (2018) Weight gain after treatment of Graves' disease in children. Clin Endocrinol 88(1):66-70

7. Conaglen HM, Tamatea J, Conaglen JV, Elston MS (2018) Treatment choice, satisfaction and quality of life in patients with Graves' disease. Clin Endocrinol 88(6):977-984
8. Ljunggren JG, Törring O, Wallin G et al (1998) Quality of life aspects and costs in treatment of Graves' hyperthyroidism with antithyroid drugs, surgery, or radioiodine: results from a prospective, randomized study. Thyroid 8(8):653-659

9. Abraham-Nordling M, Wallin G, Träisk F et al (for the Thyroid Study Group of TT 96) (2010) Thyroid-associated ophthalmopathy; quality of life follow-up of patients randomized to treatment with antithyroid drugs or radioiodine. Eur J Endocrinol 163(4):651-657

10. De la Vieja A, Santisteban P (2018) Role of iodide metabolism in physiology and cancer. Endocr-Relat Cancer 25(4):R225-R245 